\title{
$b \rightarrow d$ Penguins: CP Violation, General Lower Bounds on the Branching Ratios and Standard Model Tests
}

\author{
Robert Fleischer \\ CERN, Switzerland \\ E-mail: Robert.Fleischer@cern.ch \\ Stefan Recksiegel* \\ TU Muenchen, Germany \\ E-mail: Stefan.Recksiegel@ph.tum.de
}

\begin{abstract}
With the wealth of new data from the $B$-factories, $b \rightarrow d$ penguin decays become available for study, in addition to their $b \rightarrow s$ counterparts that have proven an indespensable tool for the exploration of new-physics effects in flavour physics. A prominent example of the $b \rightarrow d$ penguin transitions is $\bar{B}_{d}^{0} \rightarrow K^{0} \bar{K}^{0}$. We show that this decay can be charaterized in the Standard Model by a surface in the observable space of the direct and mixing-induced CP asymmetries and the branching ratio. The form of this surface, which is theoretically clean, implies a lower bound for the branching ratio that has recently been confirmed experimentally. If future measurements of the $\mathrm{CP}$ asymmetries yield a point away from the SM surface, this would be an interesting signal of new physics. We point out that the hadronic parameters in $\bar{B}_{d}^{0} \rightarrow K^{0} \bar{K}^{0}$ that parameterize the position on the SM surface are related to hadronic parameters in the $B \rightarrow \pi K$ system. The fact that the branching ratio of $\bar{B}_{d}^{0} \rightarrow K^{0} \bar{K}^{0}$ is very close to its lower bound yields interesting implications for $B \rightarrow \pi K$ even without knowledge of the CP asymmetries of $\bar{B}_{d}^{0} \rightarrow K^{0} \bar{K}^{0}$. The mechanism that produces the lower bound for $\bar{B}_{d}^{0} \rightarrow K^{0} \bar{K}^{0}$ is actually much more general; we derive lower bounds for various other $b \rightarrow d$ penguin-induced processes, including $B \rightarrow \rho \gamma$ and $B^{ \pm} \rightarrow K^{(*) \pm} K^{(*)}$. Some of these theoretical lower bounds are very close to the current experimental upper bounds.
\end{abstract}

International Europhysics Conference on High Energy Physics

July 21st - 27th 2005

Lisboa, Portugal

\footnotetext{
* Speaker.
} 


\section{Introduction}

Flavour-changing neutral-current (FCNC) processes, possible in the Standard Model (SM) only through loop diagrams, are an extremely important probe for new physics (NP). The good agreement between experiment and theory in processes induced by $b \rightarrow s$ FCNCs has already put important constraints on physics beyond the SM. Due to the excellent work of the $B$-factories, we are now entering the era where $b \rightarrow d$ penguin-induced processes - typically suppressed by a factor of 20 with respect to the corresponding $b \rightarrow s$ penguin transitions - can be used to test the SM more rigorously than it was possible before.

The flavour structure of the SM, more specifically the order of magnitude of the individual elements of the Cabibbo-Kobayashi-Maskawa (CKM) matrix, allows us to derive certain relationships between different observables in $b \rightarrow d$-induced decays, and between $b \rightarrow s$ - and $b \rightarrow d$ related observables. These relationships allow us to test the SM in those cases where the corresponding observables have already been measured and to make predictions where observations are still missing.

\section{2. $B_{d}^{0} \rightarrow K^{0} \bar{K}^{0}$ : CP Violation and the Branching Ratio}

In the $\mathrm{SM}$, we can write the amplitude for the decay $B_{d}^{0} \rightarrow K^{0} \bar{K}^{0}$ as

$$
A\left(B_{d}^{0} \rightarrow K^{0} \bar{K}^{0}\right)=\lambda_{u}^{(d)} \mathscr{P}_{u}^{K K}+\lambda_{c}^{(d)} \mathscr{P}_{c}^{K K}+\lambda_{t}^{(d)} \mathscr{P}_{t}^{K K},
$$

where the $\lambda_{q}^{(d)} \equiv V_{q d} V_{q b}^{*}$ are CKM factors, and the $\mathscr{P}_{q}^{K K}$ denote the strong amplitudes of penguin topologies with internal $q$-quark exchanges, which receive tiny contributions from coloursuppressed electroweak (EW) penguins and are fully dominated by QCD penguin processes. Eliminating $\lambda_{t}^{(d)}$ with the help of the unitarity relation $\lambda_{t}^{(d)}=-\lambda_{u}^{(d)}-\lambda_{c}^{(d)}$ of the CKM matrix, we can write the amplitude as

$$
A\left(B_{d}^{0} \rightarrow K^{0} \bar{K}^{0}\right)=\lambda^{3} A \mathscr{P}_{t c}^{K K}\left[1-\rho_{K K} e^{i \theta_{K K}} e^{i \gamma}\right],
$$

where $\mathscr{P}_{t c}^{K K} \equiv \mathscr{P}_{t}^{K K}-\mathscr{P}_{c}^{K K}$, and $\rho_{K K} e^{i \theta_{K K}}$ is a function of the $\mathscr{P}_{q}^{K K}$ that we treat as an unknown hadronic parameter.

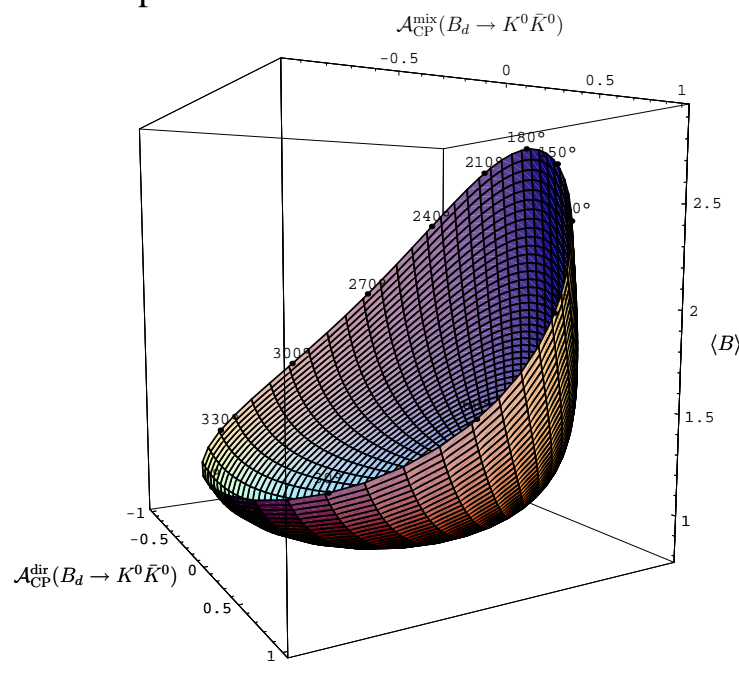

The direct and mixing-induced $\mathrm{CP}$ asymmetries $\mathscr{A}_{\mathrm{CP}}^{\mathrm{dir}}\left(B_{d} \rightarrow K^{0} \bar{K}^{0}\right)$ and $\mathscr{A}_{\mathrm{CP}}^{\mathrm{mix}}\left(B_{d} \rightarrow\right.$ $\left.K^{0} \bar{K}^{0}\right)$ are functions of only $\rho_{K K}, \theta_{K K}$, the angle $\gamma$ of the unitarity triangle, and (in the latter case) the $B_{d}^{0}-\bar{B}_{d}^{0}$ mixing phase $\phi_{d}$; the same is true for the normalized branching ratio $\langle B\rangle$, where phase-space and CKM factors as well as $\left|\mathscr{P}_{t c}^{K K}\right|^{2}$ have been factored off.

For fixed values of $\gamma$ and $\phi_{d}, \rho_{K K}$ and $\theta_{K K}$ then span a surface in the $\mathscr{A}_{\mathrm{CP}}^{\mathrm{dir}}-\mathscr{A}_{\mathrm{CP}}^{\mathrm{mix}}-\langle B\rangle$ observable space, shown in Fig. 1 for $\phi_{d}=47^{\circ}$ and $\gamma=65^{\circ}$. (The fringe is defined by $\rho_{K K}=1$, the numbers give the value for $\theta_{K K}$.)

Figure 1: The surface in observable space. 
In the SM, any measurement of the three observables has to lie on this surface, which is theoretically clean. Sufficiently accurate measurements of the branching ratio will give strong constraints on possible values for the asymmetries.

The form of the surface implies a theoretical lower bound for $\langle B\rangle$ that can be converted into a lower bound for $\mathrm{BR}\left(B_{d} \rightarrow K^{0} \bar{K}^{0}\right)$ using input from $b \rightarrow s$ penguin decays (see [1] for details). With the help of this lower bound, the recent measurement of $B_{d} \rightarrow K^{0} \bar{K}^{0}$ [3] was correctly predicted in [1]. Using the latest experimental input and the central values of the factorizable $S U(3)$-breaking parameters, we update the bound in (3) of [1] to $\operatorname{BR}\left(B_{d}^{0} \rightarrow \bar{K}^{0} K^{0}\right)>1.43_{-0.25}^{+0.17}$, nicely consistent with the old result and the recent measurements (see Table 1).

We observe that the measured $\mathrm{BR}\left(B_{d}^{0} \rightarrow \bar{K}^{0} K^{0}\right)$ is right at the lower theoretical bound (bottom of the surface in Fig. 1). This implies a value of $\rho_{K K}$ significantly different from 0 , with a small phase $\theta_{K K} ; \rho_{K K}$ can be related to a hadronic $B \rightarrow \pi K$ parameter through $\rho_{\mathrm{c}}=\varepsilon \rho_{K K}$, where $\varepsilon \equiv$ $\lambda^{2} /\left(1-\lambda^{2}\right)=0.053$. This quantity is usually neglected. However, a value of $\rho_{\mathrm{c}} \sim 0.05$, as suggested by $\operatorname{BR}\left(B_{d}^{0} \rightarrow \bar{K}^{0} K^{0}\right)$, would be rather welcome in the analysis of the $B \rightarrow \pi K$ system [4].

\section{General Lower Bounds on the Branching Ratios of $b \rightarrow d$ Penguin Processes}

The mechanism that provided the lower bound on $\operatorname{BR}\left(B_{d}^{0} \rightarrow \bar{K}^{0} K^{0}\right)$ is actually more general. We will now first use it to derive lower bounds on $b \rightarrow d \gamma$ processes, and then discuss the general $b \rightarrow d$ penguin case. The amplitude for the decay $\bar{B} \rightarrow \rho \gamma$ can be written as

$$
A(\bar{B} \rightarrow \rho \gamma)=c_{\rho} \lambda^{3} A \mathscr{P}_{t c}^{\rho \gamma}\left[1-\rho_{\rho \gamma} e^{i \theta_{\rho \gamma}} e^{-i \gamma}\right],
$$

where $c_{\rho}=1 / \sqrt{2}$ and 1 for $\rho=\rho^{0}$ and $\rho^{ \pm}$, respectively, and $A=\left|V_{c b}\right| / \lambda^{2}$. Moreover, $\mathscr{P}_{t c}^{\rho \gamma} \equiv$ $\mathscr{P}_{t}^{\rho \gamma}-\mathscr{P}_{c}^{\rho \gamma}$, where $\mathscr{P}_{t}^{\rho \gamma}$ and $\mathscr{P}_{c}^{\rho \gamma}$ are matrix elements of operators from the standard weak effective Hamiltonian (see [2] for details). $\rho_{\rho \gamma} e^{i \theta_{\rho \gamma}}$ is again a hadronic parameter that we will treat as essentially unknown. Let us now use the information offered by the $b \rightarrow s$ counterpart of our $b \rightarrow d$ transition, which is well measured and takes an amplitude of the following form:

$$
A\left(\bar{B} \rightarrow K^{*} \gamma\right)=-\frac{\lambda^{3} A \mathscr{P}_{t c}^{K^{*} \gamma}}{\sqrt{\varepsilon}}\left[1+\varepsilon \rho_{K^{*} \gamma} e^{i \theta_{K^{*}}} e^{-i \gamma}\right],
$$

where $\varepsilon$ was introduced above. The ratio of the corresponding BRs is then given by

$$
\frac{\operatorname{BR}(\bar{B} \rightarrow \rho \gamma)}{\operatorname{BR}\left(\bar{B} \rightarrow K^{*} \gamma\right)}=\varepsilon\left[\frac{\Phi_{\rho \gamma}}{\Phi_{K^{*} \gamma}}\right]\left|\frac{\mathscr{P}_{t c}^{\rho \gamma}}{\mathscr{P}_{t c}^{K^{*} \gamma}}\right|^{2} H_{K^{*} \gamma}^{\rho \gamma},
$$

where $\Phi_{\rho \gamma}$ and $\Phi_{K^{*} \gamma}$ denote phase-space factors, and

$$
H_{K^{*} \gamma}^{\rho \gamma} \equiv \frac{1-2 \rho_{\rho \gamma} \cos \theta_{\rho \gamma} \cos \gamma+\rho_{\rho \gamma}^{2}}{1+2 \varepsilon \rho_{K^{*} \gamma} \cos \theta_{K^{*} \gamma} \cos \gamma+\varepsilon^{2} \rho_{K^{*} \gamma}^{2}} .
$$

Although $\rho_{K^{*} \gamma} e^{i \theta_{K^{*} \gamma}}$ is here strongly suppressed by $\varepsilon$, we can straightforwardly include the corresponding corrections by using the flavour-symmetry relation $\rho_{\rho \gamma} e^{i \theta_{\rho \gamma}}=\rho_{K^{*} \gamma} e^{i \theta_{K^{*} \gamma}} \equiv \rho e^{i \theta}$. Treating then $(\rho, \theta)$ as completely free parameters, we can derive the following lower bound:

$$
H_{K^{*} \gamma}^{\rho \gamma} \geq\left[1-2 \varepsilon \cos ^{2} \gamma+\mathscr{O}\left(\varepsilon^{2}\right)\right] \sin ^{2} \gamma
$$




\begin{tabular}{|c|c||c|c|c|c|c|}
\hline$b \rightarrow s$ process & Exp. rate & $b \rightarrow d$ process & Naïve bound & Bound & Belle & BaBar \\
\hline$B^{+} \rightarrow K^{0} \pi^{+}$ & $24.1 \pm 1.3$ & $B_{d}^{0} \rightarrow \bar{K}^{0} K^{0}$ & $0.88_{-0.15}^{+0.11}$ & $1.43_{-0.25}^{+0.17}$ & $0.8 \pm 0.32$ & $1.19_{-0.37}^{+0.42}$ \\
\hline$B^{+} \rightarrow K^{0} \pi^{+}$ & $24.1 \pm 1.3$ & $B^{+} \rightarrow \bar{K}^{0} K^{+}$ & $1.03_{-0.14}^{+0.11}$ & $1.69_{-0.23}^{+0.19}$ & $1.0 \pm 0.41$ & $1.50 \pm 0.51$ \\
\hline$B^{+} \rightarrow K^{* 0} \pi^{+}$ & $9.7 \pm 1.2$ & $B^{+} \rightarrow \bar{K}^{* 0} K^{+}$ & $0.46_{-0.07}^{+0.06}$ & $0.76_{-0.12}^{+0.10}$ & \multicolumn{2}{|c|}{$<5.3$ (CLEO) } \\
$B^{+} \rightarrow K^{* 0} \rho^{+}$ & $10.6 \pm 1.9$ & $B^{+} \rightarrow \bar{K}^{* 0} K^{*+}$ & $0.46_{-0.10}^{+0.09}$ & $0.73_{-0.16}^{+0.15}$ & \multicolumn{2}{|c|}{$<71$ (CLEO) } \\
\hline$B_{d}^{0} \rightarrow K^{* 0} \gamma$ & $40.1 \pm 2.0$ & $B_{d}^{0} \rightarrow \rho^{0} \gamma$ & $0.86_{-0.12}^{+0.10}$ & $0.51_{-0.11}^{+0.13}$ & $1.17_{-0.32}^{+0.36}$ & $0.0 \pm 0.22$ \\
\hline$B^{+} \rightarrow K^{*+} \gamma$ & $40.3 \pm 2.6$ & $B^{+} \rightarrow \rho^{+} \gamma$ & $1.73_{-0.26}^{+0.22}$ & $1.03_{-0.23}^{+0.27}$ & $0.55_{-0.39}^{+0.45}$ & $0.9_{-0.51}^{+0.61}$ \\
\hline
\end{tabular}

Table 1: Post-LP2005: Belle and BaBar data, theory lower bounds.

which can be converted into a lower bound for $\bar{B} \rightarrow \rho \gamma$ through (3.3) and the measured value of the $\bar{B} \rightarrow K^{*} \gamma$ branching ratio.

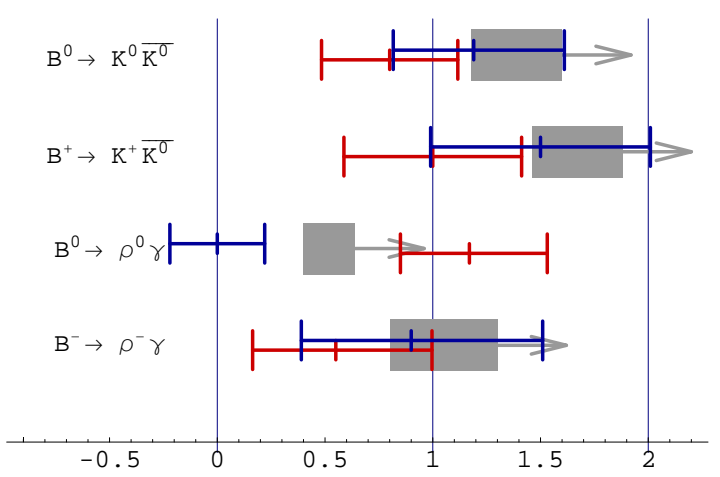

Figure 2: Experimental results (upper: BaBar, lower: Belle) and theoretical limits

Taking into account phase-space effects and factorizable $S U(3)$-breaking corrections, we obtain the lower bounds given in Table 1 . For comparision, we also show the bounds that result from neglecting the $S U(3)$-breaking corrections ("naïve bound"). The bounds are consistent with the experimental results for $B_{d}^{0} \rightarrow \rho^{0} \gamma$ and $B^{+} \rightarrow \rho^{+} \gamma$, although of course the well-known isospin-breaking puzzle of the Belle result remains.

In a similar way we can also derive theoretical lower limits for other $b \rightarrow d$ penguin decays. We list bounds for $B^{ \pm} \rightarrow K^{(*) \pm} K^{(*)}-$ together with the respective $b \rightarrow s$ decay that was used for the bound - in Table 1 (experimental data are taken from [5]); some more channels, including also $B^{ \pm} \rightarrow \pi / \rho^{ \pm} \ell^{+} \ell^{-}$modes, are discussed in [2]. For the currently most interesting decays, the theoretical predictions and measurements by BaBar and Belle are also plotted in Fig. 2. It will be interesting to confront our bounds with future data.

\section{References}

[1] R. Fleischer and S. Recksiegel, Eur. Phys. J. C 38 (2004) 251 [arXiv:hep-ph/0408016].

[2] R. Fleischer and S. Recksiegel, Phys. Rev. D 71 (2005) 051501 [arXiv:hep-ph/0409137].

[3] B. Aubert et al. [BABAR Collaboration], arXiv:hep-ex/0408080;

K. Abe et al. [BELLE Collaboration], arXiv:hep-ex/0506080.

[4] A. J. Buras, R. Fleischer, S. Recksiegel and F. Schwab, Acta Phys. Polon. B 36 (2005) 2015 [arXiv:hep-ph/0410407].

[5] http://www.slac.stanford.edu/xorg/hfag/. 